\title{
EXISTENCE AND UNIQUENESS OF POSITIVE SOLUTION OF A LOGISTIC EQUATION WITH NONLINEAR GRADIENT TERM
}

\author{
DAVID RUIZ AND ANTONIO SUÁREZ
}

\begin{abstract}
The main goal of this work is to study the existence and uniqueness of positive solution of a logistic equation including a nonlinear gradient term. In particular, we use local and global bifurcation together with some a-priori estimates. To prove uniqueness, the sweeping method of Serrin is employed.
\end{abstract}

\section{INTRODUCTION}

In this paper we study the existence and possible uniqueness of positive solutions of the problem:

$$
\begin{cases}-\Delta u=\lambda u-u^{p} \pm|\nabla u|^{q} & \text { in } \Omega, \\ u=0 & \text { on } \partial \Omega,\end{cases}
$$

where $\Omega \subset \mathbb{R}^{N}$ is a bounded and regular domain and $1<p, q$. The constant $\lambda \in \mathbb{R}$ will be regarded as a bifurcation parameter.

When the nonlinear gradient term does not appear, the above equation is the classical logistic one, which has been extensively studied in the literature. It is very well-known that there exists a positive solution if, and only if, $\lambda>\lambda_{1}$, where $\lambda_{1}$ denotes the principal eigenvalue of the laplacian. In such case, this solution is unique and stable.

However, when the gradient term is included, the equation is less known. In the particular case $\lambda=0$ and under a blow-up Dirichlet condition, it was studied in [4], [12] and [18]. In these papers, the solution is obtained as limit of a sequence of solutions of the corresponding nonhomogeneous Dirichlet problems. In each step, the authors use [17], Theorem 8.3, page 301, and so $q \leq 2$ must be imposed. Some other papers consider similar problems with critical growth $(q=2)$, in which a convenient change of variables works (see, for instance, $[20,21]$ and [23]).

2000 Mathematics Subject Classification. 35B32, 35B45, 35J25, 35J60.

Key words and phrases. Elliptic logistic equation, bifurcation, a-priori bounds, nonlinear gradient term.

The first author has been supported by the Spanish Ministry of Science and Technology under Grant BFM2002-02649 and by J. Andalucía (FQM 116). The second author has been supported by the Spanish Ministry of Science and Technology under Grant BFM200306446. 
Generally speaking, equations of the form

$$
-\Delta u=f(x, u, \nabla u) \quad \text { in } \Omega, \quad u=0 \quad \text { on } \partial \Omega,
$$

have been very studied in the literature, see the classical works $[3,5,6,8$, $10,15,22]$. Most of these are concerned with a-priori estimates, and from those they get existence by using topological methods or sub-super solution techniques.

In order to obtain our existence results, we use bifurcations methods (local and global) and a-priori bounds in $C^{1}$. It is well-known that these a-priori estimates hold for $q \leq 2$. However, as a special feature of our problem, we can even provide a $C^{1}$ estimate for $\left(P_{-}\right)$and any $q>1$. In order to do that, we first find a supersolution, greater than any solution $u$ of $\left(P_{-}\right)$, and from this we can estimate the maximum of the function $w=|\nabla u|^{2} / 2$ (see, for instance, [26], Chapter 5 and [23]).

In general, the uniqueness of elliptic equations is a hard question as was remarked in [11]. For the equation (1) one can easily obtain uniqueness from the maximum principle if $f$ is decreasing in $u$ (see [13], Theorem 8.1). Clearly, the function $f(u)=\lambda u-u^{p}-|\nabla u|^{q}$ is not decreasing in $u$ for $\lambda>0$, and these results cannot be applied. Anyway, $u=0$ is always a solution: we are looking for uniqueness of positive solutions.

We present here a result of uniqueness of positive solutions for (1) which generalizes a classical one for semilinear equations, see for instance $[2,7,14]$, [16] (Theorems 7.14, 7.15), [24] (page 39) and the references therein. Our proof makes use of the sweeping method of Serrin ([25], page 12), as in $[16,24]$. To the best of our knowledge, the uniqueness result stated in Theorem 4.1 is completely new.

Generally speaking, we show that in the case $\left(P_{-}\right)$the results about existence and uniqueness are similar to those for the semilinear equation. However, in the case $\left(P_{+}\right)$the presence of the gradient may have an important influence (depending on the values of $p$ and $q$, see Theorem 3.3). In some case there will not be uniqueness of positive solution.

An outline of the work is as follows: in Section 2 we prove the existence and non-existence results using bifurcation and a-priori estimates. In Section 3 the general result of uniqueness is established.

\section{Preliminaries}

In this section we state the exact version of the maximum principle that will be used throughout the paper. The results we present are classical: we include them for the sake of clarity.

We begin with a the generalized weak maximum principle. A more general statement and the proof can be found in [13], Theorem 8.1.

Theorem 2.1. Let $\Omega$ be a bounded smooth domain in $\mathbb{R}^{N}, c_{i}, d: \Omega \rightarrow \mathbb{R}$ be $L^{\infty}$ functions $(i=1 \ldots n), d(x) \geq 0$. Suppose that $u \in H_{0}^{1}(\Omega)$ is a weak 
solution of the inequality:

$$
-\Delta u+\sum_{i=1}^{N} c_{i}(x) u_{i}+d(x) u \geq 0
$$

Then, $u \geq 0$.

We now state a version of the strong maximum principle for $C^{1}$ weak solutions:

Theorem 2.2. Let $\Omega$ be a bounded smooth domain in $\mathbb{R}^{N}, c_{i}, d: \Omega \rightarrow \mathbb{R}$ are $L^{\infty}$ functions $(i=1 \ldots n), d(x) \geq 0$. Suppose that $u \in C^{1}(\bar{\Omega})$ is a weak solution of the inequality (2). Assume also that $u$ is not constantly equal to zero, and $u(x)=0 \forall x \in \partial \Omega$.

Then, $u(x)>0 \forall x \in \Omega$ and $\frac{\partial u}{\partial \eta}(x)<0 \forall x \in \partial \Omega$, where $\eta$ is the unit outward normal to $\partial \Omega$ at $x$.

Proof. The proof follows that of Lemma 3.4 of [13]. Since the statement we have chosen does not match exactly with that of [13], we reproduce the proof in detail.

There are several steps:

Step 1: Define $B=B(y, R) \subset \mathbb{R}^{N}$ an open ball, and suppose that $u$ verifies inequality (2) in $B, u(x)>0 \forall x \in B, u\left(x_{0}\right)=0$ for some $x_{0} \in \partial B$. We claim that $\frac{\partial u}{\partial \eta}\left(x_{0}\right)<0$, where $\eta$ is the unit outward normal to $B$ at $x_{0}$.

We can assume $y=0$. Let $\alpha>0$ be a constant to be determined later, and define $v(x)=e^{-\alpha|x|^{2}}-e^{-\alpha R^{2}}$ defined in the annulus $A=\left\{x \in \mathbb{R}^{N}\right.$ : $R / 2 \leq|x| \leq R\}$. We compute:

$$
\begin{gathered}
-\Delta v+\sum_{i=1}^{N} c_{i}(x) v_{i}+d(x) v= \\
e^{-\alpha|x|^{2}}\left(-4|x|^{2} \alpha^{2}+2 \alpha N-2 \alpha \sum_{i=1}^{N} c_{i}(x) x_{i}+d(x)\left[1-e^{-\alpha\left(R^{2}-|x|^{2}\right)}\right]\right) \geq \\
e^{-\alpha|x|^{2}}\left(-4(R / 2)^{2} \alpha^{2}+2 \alpha N-2 \alpha M R-M\right)
\end{gathered}
$$

where $M$ is a constant such that $M>\|d\|_{L^{\infty}}, M>\left(\sum_{i=1}^{N}\left\|c_{i}\right\|_{L^{\infty}}^{2}\right)^{1 / 2}$.

We now choose $\alpha$ large enough such that (3) is positive for any $x \in A$. Clearly, $v(x)=0 \leq u(x)$ for $|x|=R$. Since $u$ is positive in the ball, we can take $\varepsilon>0$ small enough such that $\varepsilon v(x)<u(x)$ for $|x|=R / 2$.

Now, apply Theorem 2.1 to the function $u-\varepsilon v$ in $A$, to conclude that $u(x) \geq \varepsilon v(x) \forall x \in A$. Recall now that $u\left(x_{0}\right)=v\left(x_{0}\right)=0$, to conclude that

$$
\frac{\partial u}{\partial \eta}\left(x_{0}\right)<\varepsilon \frac{\partial v}{\partial \eta}\left(x_{0}\right)<0
$$


Step 2: $u(x)>0$ for any $x \in \Omega$.

By Theorem 2.1, we have that $u \geq 0$. In order to prove the strict inequality, we reason by contradiction. Take $\Omega_{0}=\{x \in \Omega: u(x)=0\}$, $\Omega_{+}=\{x \in \Omega: u(x)>0\}$. Observe that both previous sets are nonempty. Choose $y \in \Omega_{+}$such that $R=d\left(y, \Omega_{0}\right)<d(y, \partial \Omega)$. Then, $B(y, R) \subset \Omega_{+}$ and there exists $x_{0} \in \partial B \cap \Omega_{0}$. Obviously $u$ attains a minimum at $x_{0}$, and hence $\nabla u\left(x_{0}\right)=0$. We arrive then to a contradiction with Step 1 .

Step 1: $\frac{\partial u}{\partial \eta}(x)<0 \forall x \in \partial \Omega$.

Take $x_{0} \in \partial \Omega$, and $B$ an interior sphere at $x_{0}$. Thanks to Step $2, u(x)>0$ for $x \in B$, and $u\left(x_{0}\right)=0$. We conclude by applying Step 1 .

In the previous theorem the condition $d(x) \geq 0$ is needed. In next Theorem we consider any $L^{\infty}$ function $d(x)$ but assuming that the function $u$ is nonnegative (which is no longer provided by Theorem 2.1).

Theorem 2.3. Let $\Omega$ be a bounded smooth domain in $\mathbb{R}^{N}$, and $c_{i}, d: \Omega \rightarrow \mathbb{R}$ are $L^{\infty}$ functions $(i=1 \ldots n)$. Suppose that $u \in C^{1}(\bar{\Omega})$ is a weak solution of the inequality (2). Assume also that $u(x) \geq 0$ but is not constantly equal to zero, and $u(x)=0 \forall x \in \partial \Omega$.

Then, $u(x)>0 \forall x \in \Omega$ and $\frac{\partial u}{\partial \eta}(x)<0 \forall x \in \partial \Omega$, where $\eta$ is the unit outward normal to $\partial \Omega$ at $x$.

Proof. We denote $d^{+}(x)=\max \{d(x), 0\}$. Since $u$ is nonnegative, it follows:

$$
-\Delta u+\sum_{i=1}^{N} c_{i}(x) u_{i}+d^{+}(x) u \geq-\Delta u+\sum_{i=1}^{N} c_{i}(x) u_{i}+d(x) u \geq 0
$$

Now we conclude the proof by applying Theorem 2.2 to the operator:

$$
L[z]=-\Delta z+\sum_{i=1}^{N} c_{i}(x) z_{i}+d^{+}(x) z
$$

\section{Bifurcation of POSITIVE SOLUtion}

First, we need some notations: $\lambda_{1}$ and $\varphi_{1}$ stand for the principal eigenvalue of $-\Delta$ subject to homogeneous Dirichlet boundary condition and its positive eigenfunction associated, respectively.

We denote by $E:=C^{1}(\bar{\Omega})$ and $P=\{u \in E: u(x) \geq 0 \forall x \in \Omega\}$ its positive cone. We look for $u$ solution belonging to $P$. Observe, that if $u \in E$ is solution of $\left(P_{ \pm}\right)$, by the elliptic regularity $u \in C^{3, \alpha}(\bar{\Omega})$ for some $\alpha \in(0,1)$. On the other hand, if $u$ is a nontrivial solution in $P$, we have that

$$
-\Delta u \mp|\nabla u|^{q-2} \nabla u \cdot \nabla u-\left(\lambda-u^{p-1}\right) u=0,
$$


and so, Theorem 2.3 implies that $u(x)>0$ for any $x \in \Omega$, and also that $\frac{\partial u}{\partial \eta}(x)<0$, where $x \in \partial \Omega$ and $\eta$ is the unit outward normal to $\partial \Omega$ at $x$. That is to say, $u \in \operatorname{int}(P)$; in such case, we will say that $u$ is positive.

Our first result provides us with the existence of a continuum of positive solutions of $\left(P_{ \pm}\right)$. We also obtain an a-priori bound in $L^{\infty}$ for any solution of $\left(P_{ \pm}\right)$in $E$.

Proposition 3.1. There exists an unbounded continuum of positive solution $\mathcal{C} \subset \mathbb{R} \times E$ bifurcating from the trivial solution at $\lambda=\lambda_{1}$.

Moreover, if $(\lambda, u)$ is a solution of $\left(P_{ \pm}\right), u \neq 0$, then

$$
\lambda>0 \text {, and }\|u\|_{\infty} \leq \lambda^{1 /(p-1)} .
$$

Proof. Observe that $\left(P_{ \pm}\right)$can be written as

$$
u=\mathcal{K}(\lambda u+\mathcal{N}(u)), \quad \text { in } E,
$$

where $\mathcal{K}:=(-\Delta)^{-1}: C(\bar{\Omega}) \rightarrow E$ under homogeneous Dirichlet boundary conditions, and:

$$
\mathcal{N}: E \rightarrow C(\bar{\Omega}), \quad \mathcal{N}(u):=\left(-u^{p} \pm|\nabla u|^{q}\right)
$$

Observe that if $f \in C(\bar{\Omega})$, then $u=\mathcal{K}(f)$ belongs to $W^{2, p}$ for all $p>1$ (see $[1]$ ), and hence $u \in C^{1, \gamma}$. Because of that, the operator $\mathcal{K}$ is compact. The maximum principle implies also that is strongly positive.

The nonlinear operator $\mathcal{N}$ is continuous and bounded. Moreover, since $p>1, q>1$, we have that $\mathcal{N}(u)=o\left(\|u\|_{E}\right)$ as $u \rightarrow 0$; we can apply for instance Theorem 6.5.5 of [19] and conclude the existence of an unbounded continuum $\mathcal{C}$ in $\mathbb{R} \times P$ of positive solution of $\left(P_{ \pm}\right)$emanating from $\left(\lambda_{1}, 0\right)$.

On the other hand, suppose that $\lambda \leq 0$ and $u \in P$ is a solution of $\left(P_{ \pm}\right)$. Then, $L[u]=0$, where $L$ is defined as:

$$
L[z]=-\Delta z \mp|\nabla u|^{q-2} \nabla u \cdot \nabla z-\left(\lambda-u^{p-1}\right) z=0,
$$

Since $\left(\lambda-u^{p-1}\right) \leq 0$, we can apply Theorem 2.1 to $-u$ to conclude that $u \leq 0$. Therefore, $u=0$.

Finally, if $x_{M} \in \Omega$ is such that $u\left(x_{M}\right)=\max _{x \in \bar{\Omega}} u(x)$, then

$$
\lambda u\left(x_{M}\right)-u\left(x_{M}\right)^{p}-\left|\nabla u\left(x_{M}\right)\right|^{q} \geq 0,
$$

and now, taking into account that $\nabla u\left(x_{M}\right)=0$, we get (4).

The next result characterizes the existence and uniqueness of positive solution of $\left(P_{-}\right)$.

Theorem 3.2. Consider the case $\left(P_{-}\right)$. For all $p, q>1$ there exists a positive solution if, and only if, $\lambda>\lambda_{1}$. Moreover, if $\lambda>\lambda_{1}$ there exists a unique positive solution of $\left(P_{-}\right)$which is linearly asymptotically stable.

Proof. If $u$ is a positive solution of $\left(P_{-}\right)$, then multiplying the equation by $\varphi_{1}$ and integrating by parts, we have

$$
\left(\lambda_{1}-\lambda\right) \int_{\Omega} u \varphi_{1}=\int\left(-u^{p}-|\nabla u|^{q}\right) \varphi_{1}
$$


which implies that $\lambda>\lambda_{1}$.

Now, by Proposition 3.1 the proof of existence for $\lambda>\lambda_{1}$ concludes if we find a priori estimates. Specifically, we claim that if $(\lambda, u)$ is a positive solution of $\left(P_{-}\right)$and $\lambda \in I \subset \mathbb{R}$ compact, then there exists $C>0$ such that

$$
\|u\|_{E} \leq C \text {. }
$$

We prove the result for all $q>1$ by estimating first the derivative in the boundary, and afterwards in the interior of $\Omega$.

First of all, we claim that if $u$ is a solution of $\left(P_{-}\right)$then

$$
u \leq \theta_{\lambda},
$$

where $\theta_{\lambda}$ is the unique positive solution of the logistic equation

$$
\begin{cases}-\Delta u=\lambda u-u^{p} & \text { in } \Omega, \\ u=0 & \text { on } \partial \Omega .\end{cases}
$$

Indeed, if $u$ is a solution of $\left(P_{-}\right)$then $u$ is a subsolution of (7). Clearly, a large positive constant is a supersolution of (7). The sub-super solution method yields a solution of (7) greater than $u$, and since $\theta_{\lambda}$ is the unique positive solution of (7), (6) is verified.

Hence, it follows that

$$
\frac{\partial \theta_{\lambda}}{\partial \eta} \leq \frac{\partial u}{\partial \eta}<0 \quad \text { on } \partial \Omega
$$

This gives us an a-priori bound of $\nabla u$ on the boundary.

In order to estimate the gradient in the interior of $\Omega$, define:

$$
w:=\frac{1}{2}|\nabla u|^{2} .
$$

It is not hard to show that

$$
\Delta w=2 w\left(p u^{p-1}-\lambda\right)+q|\nabla u|^{q-2} \nabla u \cdot \nabla w+\sum_{i, j=1}^{N}\left(\frac{\partial^{2} u}{\partial x_{i} \partial x_{j}}\right)^{2} .
$$

Assume that the maximum of $w$ is attained at $x_{M} \in \Omega$, then using that $\Delta w\left(x_{M}\right) \leq 0$ and $\nabla w\left(x_{M}\right)=0$, we get

$$
2 w\left(x_{M}\right)\left(\lambda-p u^{p-1}\left(x_{M}\right)\right) \geq \sum_{i, j=1}^{N}\left(\frac{\partial^{2} u}{\partial x_{i} \partial x_{j}}\left(x_{M}\right)\right)^{2} .
$$

On the other hand, there exists $C>0$ such that

$$
\sum_{i, j=1}^{N}\left(\frac{\partial^{2} u}{\partial x_{i} \partial x_{j}}\left(x_{M}\right)\right)^{2} \geq C\left(\Delta u\left(x_{M}\right)\right)^{2}=C\left(-\lambda u\left(x_{M}\right)+u\left(x_{M}\right)^{p}+\left|\nabla u\left(x_{M}\right)\right|^{q}\right)^{2},
$$

By taking into account the $L^{\infty}$ bound of $u$, there exist positive constants $C_{1}, C_{2}>0$ such that

$$
2 w\left(x_{M}\right)\left(\lambda-p u^{p-1}\left(x_{M}\right)\right) \geq C_{1} w\left(x_{M}\right)^{q}-C_{2} .
$$


Inequalities (8), (10) imply the a-priori estimate in $C^{1}$ of the solutions of $\left(P_{-}\right)$for any $\lambda$ fixed.

The uniqueness of positive solution follows from Theorem 4.1, whose statement and proof are postponed to the next section.

Let $u_{0}>0$ be a positive solution of $\left(P_{-}\right)$. We now plan to prove that $u_{0}$ is asymptotically stable. It is well-known (see, for instance, [24]) that it suffices to show that the first eigenvalue of the linearized around of $u_{0}$ is positive, i.e., the first eigenvalue of the following problem:

$$
\begin{cases}L[v]=\sigma v & \text { in } \Omega \\ v=0 & \text { on } \partial \Omega\end{cases}
$$

where

$$
L[v]:=-\Delta v+q\left|\nabla u_{0}\right|^{q-2} \nabla u_{0} \cdot \nabla v+\left(p u_{0}^{p-1}-\lambda\right) v .
$$

For that, it suffices to find a positive supersolution $\bar{u}$ of $L$, that is, a positive function $\bar{u}$ such that $L[\bar{u}] \geq 0$ in $\Omega$ and $\bar{u} \geq 0$ on $\partial \Omega$ with some inequality strict. Take $\bar{u}=u_{0}$, then

$$
L[\bar{u}]=(q-1)\left|\nabla u_{0}\right|^{q}+(p-1) u_{0}^{p}>0 \quad \text { in } \Omega \text { and } \bar{u}=0 \quad \text { on } \partial \Omega .
$$

The proof is completed.

Observe that for $\left(P_{-}\right)$the bifurcation is always supercritical for any value of $p$ and $q$. This is different in the case $\left(P_{+}\right)$.

Theorem 3.3. Consider the case $\left(P_{+}\right)$.

(1) With respect to the local bifurcation, we obtain:

(a) If $p>q$ (resp. $p<q$ ) the bifurcation is subcritical (resp. supercritical).

(b) If $p=q$, and $\left(\lambda_{n}, u_{n}\right)$ is a solution of $\left(P_{+}\right)$such that $\lambda_{n} \rightarrow \lambda_{1}$ and $\left\|u_{n}\right\|_{E} \rightarrow 0$ as $n \rightarrow \infty$, then

$$
0<\left(\lambda_{n}-\lambda_{1}\right)\left(\int_{\Omega} \varphi_{1}^{p+1}-\int_{\Omega}\left|\nabla \varphi_{1}\right|^{q} \varphi_{1}\right) .
$$

whenever this last term is different from zero.

(2) If $q \leq 2$, then there exists at least a positive solution for $\lambda>\lambda_{1}$.

Proof. It is not difficult to show that we are in conditions of apply the Crandall-Rabinowitz's Theorem, see [9], and so if $Y$ is any closed subspace of $E$ such that $E=\left[\operatorname{Span} \varphi_{1}\right] \oplus Y$, there exist $\varepsilon>0$ and two continuous functions

$$
\lambda:(-\varepsilon, \varepsilon) \mapsto \mathbb{R} \quad \text { and } \quad u:(-\varepsilon, \varepsilon) \mapsto Y
$$

with

$$
\lambda(s)=\lambda_{1}+\mu(s), \quad u(s)=s\left(\varphi_{1}+v(s)\right), \quad s \in(-\varepsilon, \varepsilon),
$$

and $\mu(0)=v(0)=0$ and in a neighborhood of $\left(\lambda_{1}, 0\right)$ all the solutions are of the form $(\lambda(s), u(s))$. Introducing this expression in the equation $\left(P_{+}\right)$and 
applying the Fredholm alternative, we get

$$
\mu(s)=\frac{s^{p-1} \int_{\Omega}\left(\varphi_{1}+v(s)\right)^{p} \varphi_{1}-s^{q-1} \int_{\Omega}\left(\nabla \varphi_{1}+\nabla v(s)\right)^{q} \varphi_{1}}{\int_{\Omega}\left(\varphi_{1}+v(s)\right) \varphi_{1}},
$$

whence we deduce the paragraph (1).

In order to prove paragraph $(2)$, recall that $\left(P_{+}\right)$has no solutions different from zero if $\lambda \leq 0$ (Theorem 3.1).

Moreover, since $q \leq 2$ the nonlinearity $f(x, \xi, \eta)=\lambda \xi-\xi^{p}+|\eta|^{q}$ satisfies that

$$
|f(x, \xi, \eta)| \leq c(|\xi|)\left(1+|\eta|^{2}\right) \quad \text { for }(x, \xi, \eta) \in \bar{\Omega} \times \mathbb{R} \times \mathbb{R}^{N},
$$

and so, by Proposition 2 of [3], it follows that

$$
\|u\|_{E} \leq \gamma\left(\|u\|_{\infty}\right) \leq C
$$

where $\gamma: \mathbb{R}_{+} \mapsto \mathbb{R}$ is an increasing function and $C$ a positive constant. Thus, the result follows by Proposition 3.1 and the fact that there exist no solution for $\lambda \leq 0$, see (4).

Corollary 3.4. If $q \leq 2$ and $p>q$, there exist at least two positive solutions of $\left(P_{+}\right)$in $\left(\lambda_{1}-\delta, \lambda_{1}\right)$ for some $\delta>0$ small.

Proof. Define $\mathcal{C} \subset \mathbb{R}^{+} \times \operatorname{int}(P)$ the connected set of solutions bifurcating from $\left(\lambda_{1}, 0\right)$. As we mentioned in the proof of Theorem 3.3, in a neighborhood $\mathcal{U}$ of $\left(\lambda_{1}, 0\right)$ all solutions of $\left(P_{+}\right)$are of the form $(\lambda(s), u(s))$ where $\lambda(s)<\lambda_{1}$ and $u(s)$ are defined in (12). We can assume that this neighborhood is given by:

$$
\mathcal{U}=\left(\lambda_{1}-\delta, \lambda_{1}+\delta\right) \times(B(0, \varepsilon) \cap P)
$$

for some $\varepsilon>0, \delta>0$. By taking smaller $\delta$ and $\varepsilon$ if necessary, we can assume that $\left(P_{+}\right)$has no solutions $(\lambda, u)$ such that $\lambda \in\left(\lambda_{1}-\delta, \lambda_{1}+\delta\right),\|u\|=\varepsilon$.

Recall that $\mathcal{C}$ does not cross the $\lambda=0$ line, and cannot blow up for finite $\lambda$, since we got a priori estimates. Then, the unbounded continuum $\mathcal{C}$ has an unbounded projection $[\underline{\lambda},+\infty)$ on the real $\lambda$-axis with $\underline{\lambda}>0$.

Reasoning by contradiction, suppose that there exists $\delta_{0} \in(0, \delta)$ such that $\left(P_{+}\right)$has a unique positive solution for $\lambda=\lambda_{1}-\delta$. Define:

$$
\begin{gathered}
\mathcal{S}_{1}=\left\{(\lambda, u) \in \mathbb{R}^{+} \times P: \lambda \in\left[\lambda_{1}-\delta_{0}, \lambda_{1}\right],\|u\|_{E}=\varepsilon\right\} \\
\mathcal{S}_{2}=\left\{(\lambda, u) \in \mathbb{R}^{+} \times P-\{0\}: \lambda=\lambda_{1},\|u\|_{E} \leq \varepsilon\right\} \\
\mathcal{S}_{3}=\left\{(\lambda, u) \in \mathbb{R}^{+} \times P: \lambda=\lambda_{1}-\delta_{0},\|u\|_{E} \geq \varepsilon\right\} \\
\mathcal{S}=\mathcal{S}_{1} \cup \mathcal{S}_{2} \cup \mathcal{S}_{3}
\end{gathered}
$$

Clearly, $S$ separates $\mathbb{R}^{+} \times P-\{0\}$ in two connected components, and $\mathcal{C} \cap \mathcal{S}=$ $\emptyset$.

Moreover, we have that the branch $(\lambda(s), u(s))$ is in one of these components, whereas any solution $(\lambda, u) \in \mathcal{C}$ with $\lambda>\lambda_{1}$ is in the other one. This is a contradiction with the fact that $\mathcal{C}$ is connected. 


\section{A uniqueness Result}

In this section we prove the uniqueness of positive solution of (1) under a certain condition on $f$, extending the result of $[2,7,14,16]$ to the case of quasilinear equations.

Theorem 4.1. Consider the problem:

$$
-\Delta u=f(x, u, \nabla u) \quad \text { in } \Omega, \quad u=0 \quad \text { on } \partial \Omega,
$$

where $f: \bar{\Omega} \times \mathbb{R}_{0}^{+} \times \mathbb{R}^{n} \rightarrow \mathbb{R}$ is a continuous function, locally Lipschitz with respect to $(u, \eta) \in \mathbb{R}_{0}^{+} \times \mathbb{R}^{n}$. Suppose that $f$ verifies also the following condition:

$$
\left.\begin{array}{l}
\text { For any } x \in \Omega,(u, \eta) \in \mathbb{R}^{+} \times \mathbb{R}^{n} \text {, the function } \\
t \mapsto \frac{f(x, t u, t \eta)}{t} \text { is strictly decreasing in } t \in \mathbb{R}^{+}
\end{array}\right]
$$

Then, there exists at most one non-negative nonzero solution of problem (13) in $E$.

Proof. First, let us show that (14) implies that $f(x, 0,0) \geq 0$ for any $x \in \Omega$. Otherwise, if there exists $x_{0} \in \Omega$ such that $f\left(x_{0}, 0,0\right)<0$, we would have that:

$$
\lim _{t \rightarrow 0^{+}} \frac{f\left(x_{0}, t u, t \eta\right)}{t}=-\infty
$$

for any $(u, \eta) \in \mathbb{R}^{+} \times \mathbb{R}^{n}$. This would contradict (14).

We now prove that any non-negative nonzero solution of (13) must be positive, that is, must belong to $\operatorname{int}(P)$. Given any $u \in P$ a solution, we can write:

$$
\begin{aligned}
& -\Delta u=f(x, u, \nabla u)-f(x, u, 0)+f(x, u, 0)-f(x, 0,0)+f(x, 0,0)= \\
& \frac{f(x, u, \nabla u)-f(x, u, 0)}{|\nabla u|^{2}} \nabla u \cdot \nabla u+\frac{f(x, u, 0)-f(x, 0,0)}{u} u+f(x, 0,0) .
\end{aligned}
$$

Since $u, \nabla u$ are both bounded and $f$ is locally Lipschitz, the functions:

$$
\frac{f(x, u, \nabla u)-f(x, u, 0)}{|\nabla u|^{2}} \nabla u, \frac{f(x, u, 0)-f(x, 0,0)}{u}
$$

are uniformly bounded. Therefore, $u$ is a solution of the problem $L[u]=$ $f(x, 0,0)$, where $L$ is a linear operator defined as:

$$
L[z]=-\Delta z-\frac{f(x, u, \nabla u)-f(x, u, 0)}{|\nabla u|^{2}} \nabla u \cdot \nabla z-\frac{f(x, u, 0)-f(x, 0,0)}{u} z .
$$

Recall now that $u$ vanishes in $\partial \Omega$ and is nonnegative and different from zero. Theorem 2.3 implies that $u \in \operatorname{int}(P)$.

We now prove uniqueness by using the sweeping method of Serrin. Suppose that $u, v$ are two nonnegative solutions of (13), both different from zero. By direct computation (using (14)) we conclude that the functions $s u$, with $s \in(0,1)$, are strict subsolutions of (13). Define the set:

$$
D=\{s \in[0,1]: s u(x) \leq v(x) \text { for any } x \in \Omega\}
$$


From the definition, it is obvious that $D$ is closed. Since $u, v \in \operatorname{int}(P)$, there exists $\varepsilon>0$ belonging to $D$. Take $\gamma=\max D>0$ : we claim that $\gamma=1$. Reasoning by contradiction, suppose that $\gamma \in(0,1)$. Let us call $w=\gamma u$, $w \leq v$, and recall that $w$ is a subsolution of (13).

Then, we have that:

$$
\begin{gathered}
-\Delta(v-w) \geq f(x, v, \nabla v)-f(x, w, \nabla w)= \\
f(x, v, \nabla v)-f(x, v, \nabla w)+f(x, v, \nabla w)-f(x, w, \nabla w)= \\
\frac{f(x, v, \nabla v)-f(x, v, \nabla w)}{|\nabla v-\nabla w|^{2}} \nabla(v-w) \cdot \nabla(v-w)+\frac{f(x, v, \nabla w)-f(x, w, \nabla w)}{v-w}(v-w)
\end{gathered}
$$

We now argue as above: since $u, v$ belong to $E$, the functions

$$
\frac{f(x, v, \nabla v)-f(x, v, \nabla w)}{|\nabla v-\nabla w|^{2}} \nabla(v-w), \frac{f(x, v, \nabla w)-f(x, w, \nabla w)}{v-w}
$$

are uniformly bounded. Therefore, we obtain that $\bar{L}[v-w] \geq 0$, where $\bar{L}$ is defined as

$\bar{L}[z]=-\Delta z-\frac{f(x, v, \nabla v)-f(x, v, \nabla w)}{|\nabla v-\nabla w|^{2}} \nabla(v-w) \cdot \nabla z-\frac{f(x, v, \nabla w)-f(x, w, \nabla w)}{v-w} z$

Recall now that $v-w$ vanishes in the boundary and $v-w \geq 0$. Therefore, the maximum principle (Theorem 2.3) yields that either $v-w=0$ or $v-w \in$ $\operatorname{int}(P)$. Observe now that the first possibility does not hold since $v$ is a solution of (13) and $w=\gamma u$ is not. Therefore, $v-\gamma u \in \operatorname{int}(P)$. However, this implies that $v-(\gamma+\varepsilon) u \geq 0$ for $\varepsilon>0$ small enough. Thus, $\gamma+\varepsilon \in D$, contradicting the definition of $\gamma$.

Then, $\gamma=1 \in D$, that is, $u \leq v$. We can also apply the preceding argument but changing the roles of $u$ and $v$, to obtain that $v \leq u$. This concludes the proof.

Remark 4.2. In the case in which $f$ does not depend on $\nabla u$, condition (14) is the same as the one given in $[7,16]$.

Remark 4.3. Condition (14) can be relaxed in different ways. For instance, Theorem 4.1 is also true if (14) is replaced with:

$$
\left.\begin{array}{l}
\text { For any }(u, \eta) \in \mathbb{R}^{+} \times \mathbb{R}^{n} \text {, the function } \\
t \mapsto \frac{f(x, t u, t \eta)}{t} \text { is decreasing for any } x \in \Omega \\
t \mapsto \frac{f(x, t u, t \eta)}{t} \text { is strictly decreasing for any } x \in \Omega^{\prime}
\end{array}\right]
$$

where $\Omega^{\prime} \subset \Omega$ is a subset with nonzero measure. 
Furthermore, if (14) is relaxed to

$$
\begin{aligned}
& \text { For any } x \in \Omega,(u, \eta) \in \mathbb{R}^{+} \times \mathbb{R}^{n}, \text { the function } \\
& t \mapsto \frac{f(x, t u, t \eta)}{t} \text { is decreasing in } t \in \mathbb{R}^{+}
\end{aligned}
$$

then the arguments exposed in the proof of Theorem 4.1 imply the following result:

Suppose that $u, v$ are nonnegative solutions of (13) in E, and assume that (16) holds. Then, $u$ and $v$ are proportional functions.

Acknowledgements: This work has been partially carried out during a stay of the first author in the University of Sevilla. The first author thanks the Department of Differential Equations and Numerical Analysis for their invitation and for the warm hospitality.

\section{REFERENCES}

[1] S. Agmon, A. Douglis and L. Nirenberg, Estimates near the boundary for solutions of elliptic partial differential equations satisfying general boundary conditions I, Comm. Pure Appl. Math. 12 (1959), 623-727.

[2] H. Amann, On the existence of positive solutions of nonlinear ellptic boundary value problems, Indiana Univ. Math. J. 21 (1971), 125-146.

[3] H. Amann and M. Crandall, On some existence theorems for semilinear equations, Indiana Univ. Math. J. 27 (1978), 779-790.

[4] C. Bandle and E. Giarrusso, Boundary blow up for semilinear elliptic equations with nonlinear gradient terms, Adv. Differential Equations 1 (1996), 133-150.

[5] L. Boccardo, F. Murat and J.-P. Puel, Existence de solutions faibles pour des equations elliptiques quasi-linéaires à croissance quadratique, Nonlinear Partial Differential Equations and their Applications. Collège de France Seminar, Vol. IV (Paris, 1981/1982), 19-73. Research Notes in Math. 84, Pitman, Boston, Mass.-London, 1983.

[6] L. Boccardo, F. Murat and J.-P. Puel, Rèsultats d'existence pour certains problèmes elliptiques quasilinéaires, Ann. Scuola Norm. Sup. Pisa, Cl. Sci. (4), 11 (1984), 213235.

[7] H. Brezis and L. Oswald, Remarks on sublinear elliptic equations, Nonlinear Anal. 10 (1986), 55-64.

[8] Y. Choquet-Bruhat and J. Leray, Sur le problème de Dirichlet, quasilinéaire, d'ordre 2, C. R. Acad. Sc. Paris, Ser. A. 265 (1972), 81-85.

[9] M. G. Crandall and P. H. Rabinowitz, Bifurcation from simple eigenvalues, J. Func. Anal., 8 (1971) 321-340.

[10] J. Deuel and P. Hess, A criterion for the existence of solutions of nonlinear elliptic boundary value problems, Proc. Royal Soc. Edinburgh Sect. A 74 (1974/75), 49-54.

[11] D. De Figueiredo, M Girardi and M. Matzeu, Semilinear elliptic equations with dependence on the gradient via mountain-pass techniques, Diff. and Int. Equations, 17 (2004), 119-126.

[12] E. Giarrusso, On blow up solutions of a quasilinear elliptic equation, Math. Nachr., 213 (2000), 89-104.

[13] D. Gilbarg and N. Trudinger, Elliptic Partial Differential Equations of Second Order, Springer-Verlag, Heidelberg, 1977.

[14] P. Hess, On uniqueness of positive solutions of nonlinear elliptic boundary value problems, Math. Z. 154 (1977), 17-18.

[15] J. L. Kazdan and R. J. Kramer, Invariant criteria for existence of solutions to secondorder quasilinear equations, Comm. Pure Appl. Math. 29 (1978), 619-645. 
[16] M. A. Krasnoselskii, Positive Solutions of Operator Equations, Nordhoff, Groningen (1964).

[17] O. A. Ladyzhenskaya and N. N. Uraltceva, Linear and Quasilinear Elliptic Equationsr, Academic Press, London, 1968.

[18] A. V. Lair and A. W. Wood, Large solutions of semilinear elliptic equations with nonlinear gradient terms, Int. J. Math. Math. Sci., 22 (1999), 869-883.

[19] J. López-Gómez, Spectral Theory and Nonlinear Functional Analysis, Research Notes in Mathematics 426, CRC Press, Boca Raton, 2001.

[20] M. Montenegro and M. Montenegro, Existence and nonexistence of solutions for quasilinear elliptic equations, J. Math. Anal. Appl. 245 (2000), 303-316

[21] L. Orsina and J.-P. Puel, Positive solutions for a class of nonlinear elliptic problems involving quasilinear and semilinear terms, Comm. Partial. Diff. Eq. 26 (2001), 16651689.

[22] S. I. Pohozaev, Equations of the type $\Delta u f(x, u, D u)$, Mat. Sbornik 113 (155) (1980) 324-338, 351.

[23] P. Quittner, On global existence and stationary solutions for two classes of semilinear parabolic problems, Comment. Math. Univ. Carolin. 34 (1993), 105-124.

[24] D. H. Sattinger, Topics in Stability and Bifurcation Theory, Lectures Notes in Math. 309, Berling-Heidelberg-New York, Springer, 1973.

[25] J. Serrin, Nonlinear Elliptic Equations of Second Order, AMS Symposium in Partial Differential Equations, Berkeley, August 1971.

[26] R. Sperb, Maximum Principles and Their Applications, Mathematics in Science and Engineering Vol. 157, Academic Press, New York-London, 1981.

David Ruiz, Dpto. Análisis Matemático, Univ. Granada, 18071 Granada, SPAIN

Antonio Suárez, Dpto. Ecuaciones Diferenciales y Análisis Numérico Fac. Matemáticas, C/ Tarfia s/n, 41012, Univ. Sevilla, Spain.

E-mail address: daruiz@ugr.es, suarez@us.es 